\title{
Micro-machining Characteristics of Ceramics by Harmonics of Nd:YAG Laser
}

\author{
Hikaru NAKAMURA $^{1}$, Yasuhiro OKAMOTO ${ }^{2}$ and Yoshiyuki UNO $^{3}$ \\ ${ }^{1}$ Okayama University, 3-1-1 Tsushimanaka, Okayama, 700-8530, Japan \\ E-mail: nakamura@ntmlab.mech.okayama-u.ac.jp \\ ${ }^{2}$ Ditto, E-mail: okamoto@mech.okayama-u.ac.jp \\ ${ }^{3}$ Ditto, E-mail: uno@mech.okayama-u.ac.jp
}

\begin{abstract}
:
Effect of wavelength on machining characteristics of $\mathrm{AlN}$ and $\mathrm{SiC}$ in micro-drilling using Q-swithched YAG harmonic was experimentally investigated. The drilled depth increased with the decrease of wavelength. Besides, under the reduced pressure condition, the drilled depth increased and the better surface integrity could be obtained compared to the atmospheric condition. The circumference region around drilled hole was oxidized, and the oxidization in the case of fourth harmonic was more remarkable than that in second harmonic. In the initial stage of drilling for AlN, the thermal expansion was observed at the irradiated point in the case of fundamental wavelength, while higher harmonic made it possible to drill without thermal expansion.
\end{abstract}

Keywords: Harmonics of Nd:YAG laser, Micro-drilling, Removal depth, Ceramics

\section{Introduction}

AlN and SiC have played an important role as an electronic industrial material because of its high heat conductivity and high electrical insulation [1-2]. However, it is difficult to machine accurately by conventional mechanical machining methods, since $\mathrm{AlN}$ and $\mathrm{SiC}$ as well as other ceramics are hard and brittle. On the other hand, laser beam machining makes it possible to machine materials regardless of its hardness. However, in the case of fundamental wavelength of $\mathrm{Nd}$ :YAG laser, heat affected zone, dross and thermal crack might remain on machined surface, since the process is mainly progressed by the thermal removal [3]. Photon energy becomes higher with decreasing the wavelength of laser beam. Thus, it is considered that precision micro-machining with low heat input can be expected because of its high photon energy, since the material is mainly removed by photochemical reaction in the case of short wavelength laser beam, which is generated by the wavelength conversion using nonlinear optical crystals [4]. Therefore, the fundamental machining characteristics of AlN and $\mathrm{SiC}$ by the harmonics of Nd:YAG laser, where the fundamental wavelength $\left(\lambda_{1}=1064 \mathrm{~nm}\right)$ of $\mathrm{Nd}$ :YAG laser is converted into the second harmonic $\left(\lambda_{2}=532\right.$ $\mathrm{nm})$, the third harmonic $\left(\lambda_{3}=355 \mathrm{~nm}\right)$, the fourth harmonic $\left(\lambda_{4}=266 \mathrm{~nm}\right)$ and the fifth harmonic $\left(\lambda_{5}=\right.$ $213 \mathrm{~nm}$ ), were experimentally investigated. The element analysis on the surface was carried out by using X-ray micro-analyzer in order to investigate influences of processing atmosphere on the element distribution of ceramics surface. Besides, the initial process of boring for AlN was observed, and the difference of plasma generation during laser irradiation was also discussed under atmosphere and vacuum condition.

\section{Laser irradiation method}

Figure 1 shows the schematic diagram of optical setup. The Q-switched Nd:YAG laser (QUANTEL, Brilliant) pumped by flash lamp was used in this study. Fundamental wavelength of $1064 \mathrm{~nm}$ in wavelength,

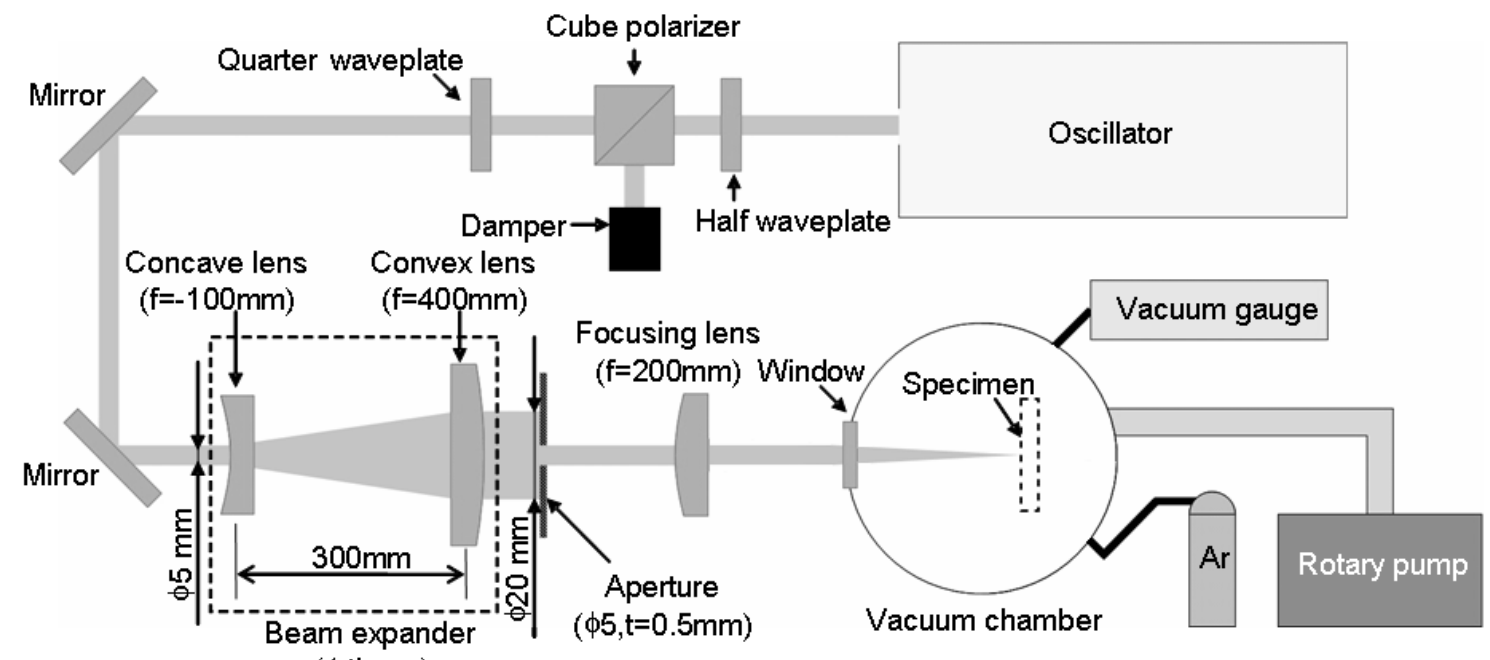

(4 times)

Figure 1: Optical setup 


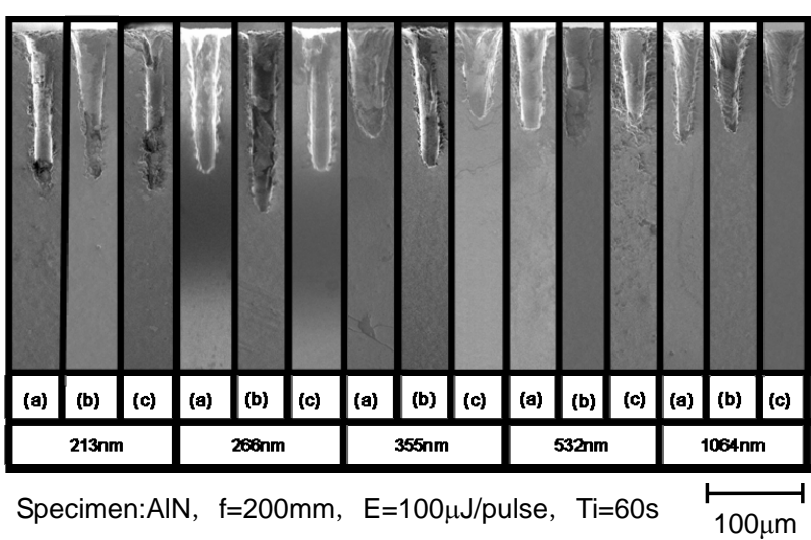

$\begin{array}{lll}\text { (a) }: 6 \times 10^{1} \mathrm{~Pa} & \text { (b) }: 1 \times 10^{2} \mathrm{~Pa} & \text { (c) }: 1 \times 10^{5} \mathrm{~Pa}\end{array}$

Figure 2: Blind hole of AlN for various wavelengths under atmospheric and reduced pressure conditions

second harmonic of $532 \mathrm{~nm}$ in wavelength, third harmonic of $355 \mathrm{~nm}$ in wavelength, fourth harmonic of $266 \mathrm{~nm}$ in wavelength and fifth harmonic of $213 \mathrm{~nm}$ in wavelength are obtained from this oscillator. The pulse frequency is kept constant at $20 \mathrm{~Hz}$, and the pulse width is $5 \mathrm{~ns}$ for every wavelength. Intensity distribution of laser beam from the oscillator was not good for the experimental processing. Thus, in order to improve the intensity distribution of laser beam from oscillator, the laser beam was expanded by 4 times using Galileo type expander, which was consist of the concave lens ( $f=100$ $\mathrm{mm}$ ) and the convex lens ( $\mathrm{f}=400 \mathrm{~mm}$ ). Then, expanded laser beam was formed by the circle mask of $5 \mathrm{~mm}$ in diameter $(\mathrm{t}=0.5 \mathrm{~mm})$. The formed laser beam was focused on the specimen surface by a lens of $200 \mathrm{~mm}$ in focal length.

In order to investigate to the effect of the processing atmosphere between the atmospheric pressure and reduced pressure conditions, the pressure in vacuum chamber was controlled to $6.0 \times 10^{1} \mathrm{~Pa}$ and $1.0 \times 10^{2} \mathrm{~Pa}$ by using the rotary pump and the argon gas The shaped laser beam was focused the on specimen set in vacuum chamber by the lens of $200 \mathrm{~mm}$ in focal length under atmospheric and reduced pressure conditions.

\section{Experiment results and discussions}

\subsection{Influence of surrounding pressure in processing}

Figure 2 shows the sectional view of blind hole of AlN for various wavelengths, when the laser beam was irradiated for $60 \mathrm{~s}$ under $100 \mu \mathrm{J}$ pulse energy condition. Figure 3 is the experimental results of $\mathrm{SiC}$ under the same irradiation condition of AlN. The drilled depths of both specimens increase with decreasing the wavelength. It is considered that the phenomenon is influenced by the difference of processing between the thermal removal and the ablation. Since the laser beam can be focused in very small spot at the focusing point, which makes it possible to become the energy density very high. Thus, the specimen was melted and evaporated immediately in the case of thermal processing by using long wavelength. However, since the power density reduces with the

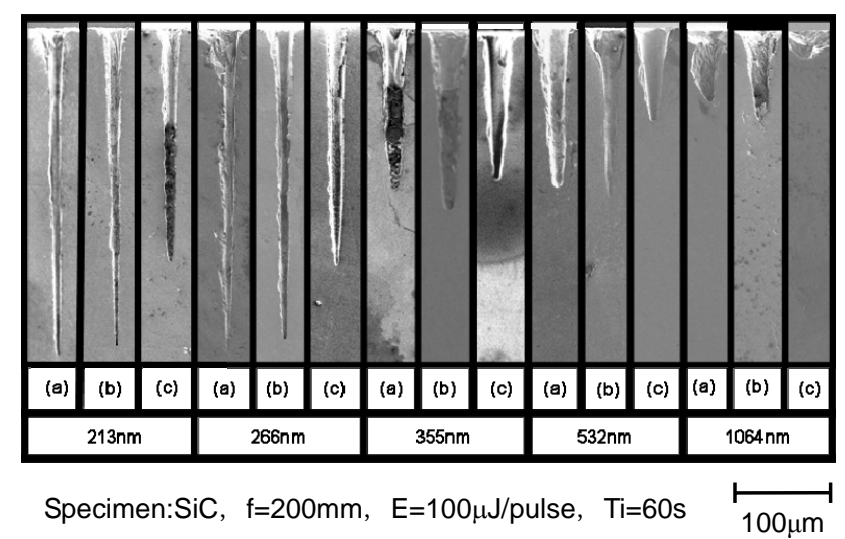
(a) $: 6 \times 10^{1} \mathrm{~Pa}$
(b) $: 1 \times 10^{2} \mathrm{~Pa}$
(c) $: 1 \times 10^{5} \mathrm{~Pa}$

Figure 3: Blind hole of SiC for various wavelengths under atmospheric and reduced pressure conditions

progress of processing, the surface temperature at the processing position could not increase up to the melting point of specimen easily. On the other hand, since the photon energy is higher in the case of shorter wavelength, the removal would be progressed by the photochemical reaction, in which the photon energy (337.0 kJ/mol @ 355 nm, 449.7 kJ/mol @266 nm, 561.6 kJ/mol @213 nm) is larger than the bonding energy of AlN (240.5 kJ/mol). Under this condition, the material can be removed, even if the processing point shifts from the focusing point. Thus, it is considered that the drilled depth with shorter wavelength became deeper than that with longer wavelength such as the fundamental wavelength

Drilled depth of $\mathrm{SiC}$ is larger than that of AlN as shown from these figures. The dissociation energy of the SiC $(111.9 \mathrm{~kJ} / \mathrm{mol})$ is smaller than that of the AlN. Therefore, it is considered that the drilled depth of $\mathrm{SiC}$ becomes deeper than that of AlN. Besides, the drilled depths of both SiC and AlN are larger than those of the atmospheric condition for every wavelength under the reduced pressure condition. Since the low pressure might make it possible to reduce the plasma generation as mentioned later, it is considered that the debries could be spouted out easily from the drilled hole under the reduced pressure condition compared to the atmospheric pressure condition.

\subsection{Element analysis around drilled hole}

SiC specimens were irradiated for $60 \mathrm{~s}$ under the atmospheric and the reduced pressure condition by second and fourth harmonic. The focusing position is set just on the surface of specimen and the pulse energy is $100 \mu \mathrm{J}$ for every case. Figure 4 and 5 show the analysis results of circumference region the drilled hole were measured by X-ray micro analyzer. The specimen surface around the drilled hole was strongly oxidized by fourth harmonic compared to second harmonic. Shorter wavelength of fourth harmonic makes it possible to dissociate the specimen to silicon and carbon strongly compared to longer wavelength of second harmonic. Therefore, it is considered that the silicon was attached to 


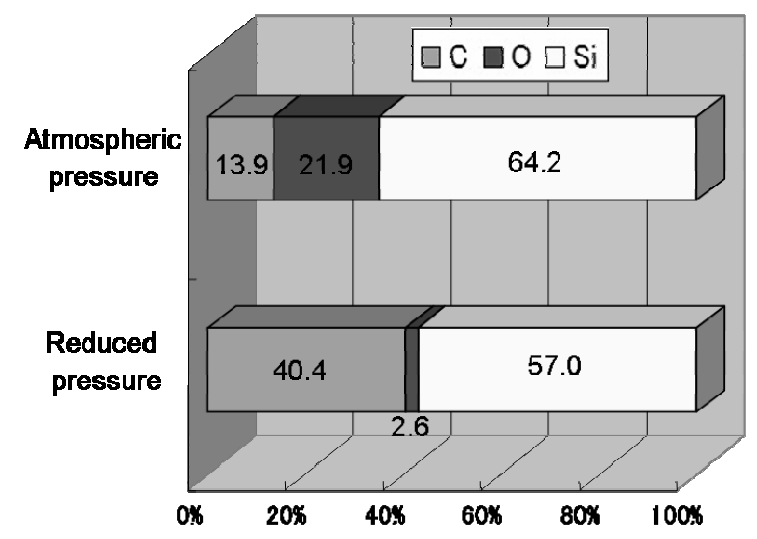

Figure 4: Quantitative analysis at circumference of drilled hole by $532 \mathrm{~nm}$ under atmospheric and reduced pressure conditions

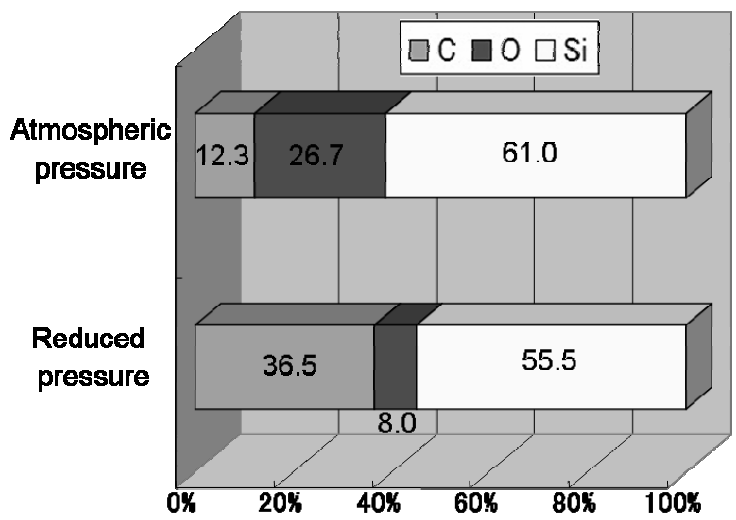

Figure 5: Quantitative analysis at circumference of drilled hole by $266 \mathrm{~nm}$ under atmospheric and reduced pressure conditions.

the oxygen in atmosphere, because the silicon is easy to bond with the oxygen rather than the carbon. Besides, the element analysis made it clear that the very close region to drilled hole was hardly oxidized. It is considered that the new processed surface is always generated at the very close region of drilled hole by the every laser irradiation. Thus, the oxidation was not heavy at the close region around drilled hole. Therefore, it is considered that the oxidation of specimen surface would be caused by the heat input from the laser induced plasma.

\subsection{Influence of plasma under the atmospheric and the reduced pressure condition}

The surface structure around the drilled hole was different between atmospheric and the reduced pressure condition as shown in Fig. 6, where SiC was irradiated for $60 \mathrm{~s}$ under the atmospheric and under the reduced pressure condition by second harmonic. The surface integrity under reduced pressure condition was superior to that under the atmospheric pressure condition. It is reported that the plasma is generated in the irradiation part when the laser beam is irradiated to the material. Therefore, it is considered the plasma generation in atmosphere might influence the surface integrity. Thus the influence of plasma generation was investigated with an ultra-high speed camera.

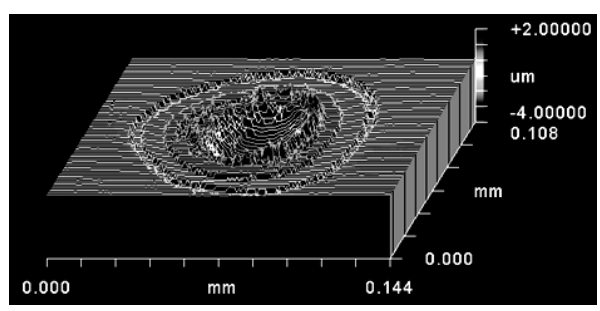

(a) $1.0 \times 10^{5} \mathrm{~Pa}$

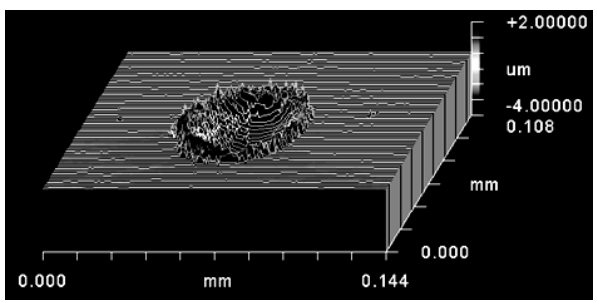

(b) $6.0 \times 10^{1} \mathrm{~Pa}$

Figure 6: 3D-images of irradiated area by $532 \mathrm{~nm}$

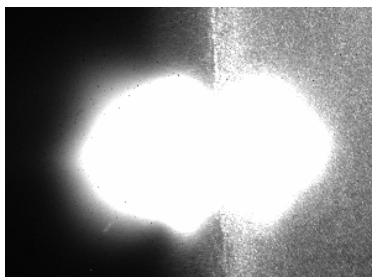

(a) $1.0 \times 10^{5} \mathrm{~Pa}$

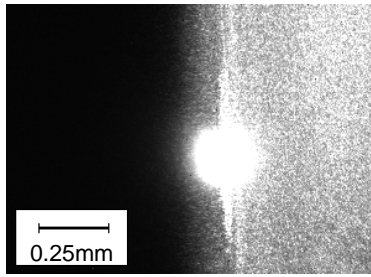

(b) $6.0 \times 10^{1} \mathrm{~Pa}$
Figure 7: Photographs of plasma under atmospheric and reduced pressure conditions

Figure 7 is the observation result of plasma by the ultra-high speed camera. The expanded area of plasma under atmospheric pressure condition is larger than the under reduced pressures condition. Therefore it is considered that the surface of specimen was improved under the reduced pressure condition.

\subsection{Difference of initial process in boring by wavelength}

Figure 8 is the measurement results of surface shape around the irradiated point at the number of pulses 5,10 , 15, 20 by fundamental wavelength under atmospheric pressure condition for AlN. The surface of the AlN started expanding a little at 5 pulses, and upheaved like the cliff at 10 pulses. The hole was generated at the center of upheaval region at 15 pulses, and its hole became larger at 20 pulses. Therefore, in the case of fundamental wavelength, the specimen was gradually expanded with increasing the number of pulses at the early stage of the processing, and then it is partly removed. And finally, the hole is formed.

Figure 9 is the measurement results of surface shape around irradiated point at the number of pulses 5, 10, 15, 20 by fourth harmonic for of AlN. In different from the case of fundamental wavelength, the hole has been already formed at 5 pulses without expansion of specimen, and the drilled depth increases with increasing the number of pulses. From these results, it is considered that the processing hole formation mechanism at the early stage is greatly different by the wavelength. 


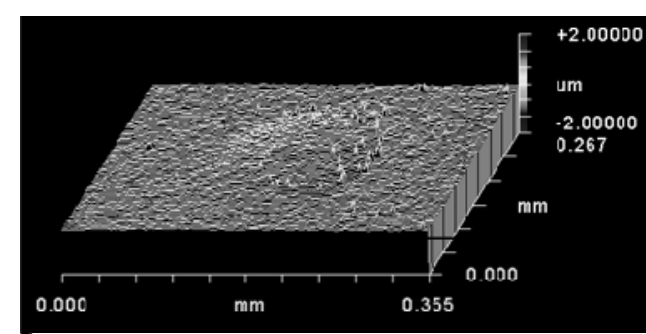

(a) 5 pulses

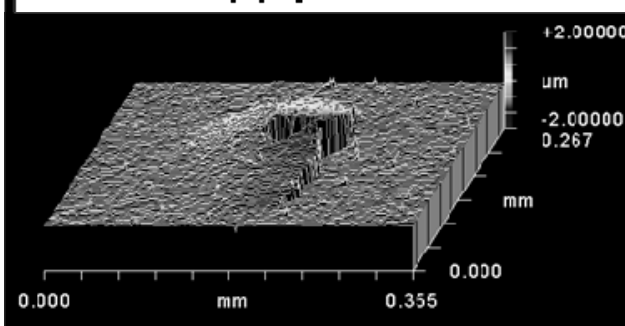

(c) 15pulses

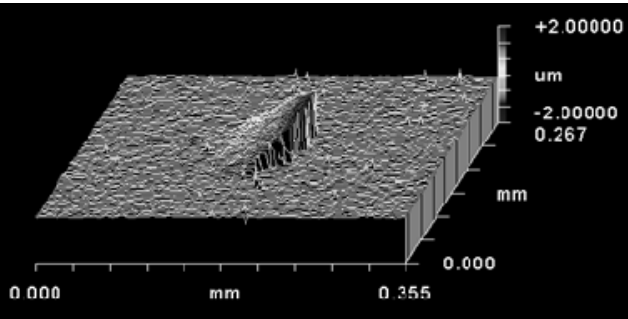

(b)10pulses

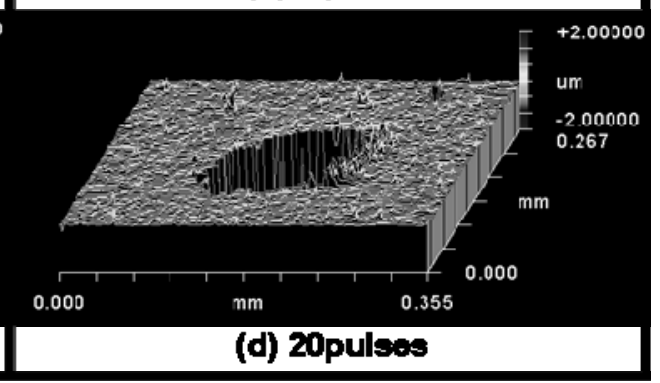

Figure 8: Changes of 3D-image at irradiated area by 1064 nm

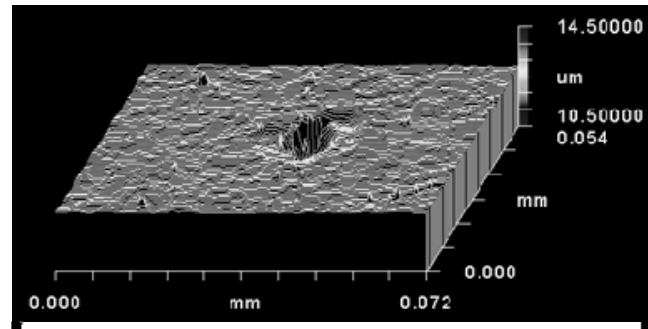

(a) Spulses

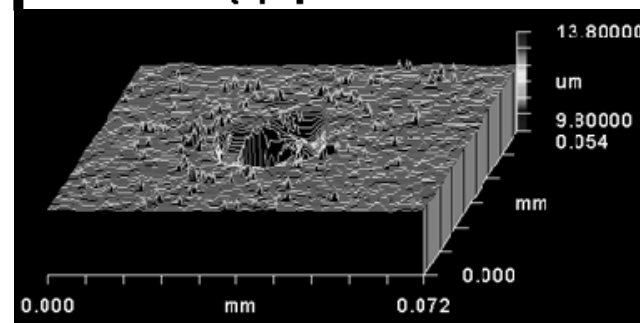

(c) 15pulses

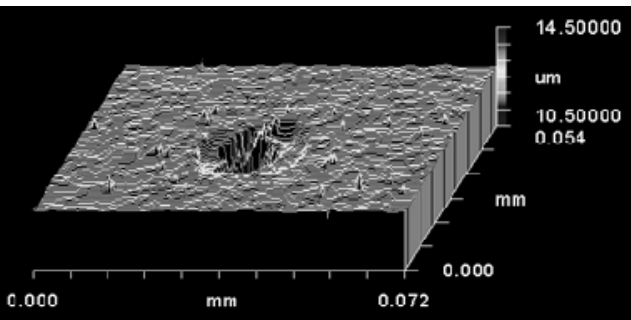

(b) 10pulses

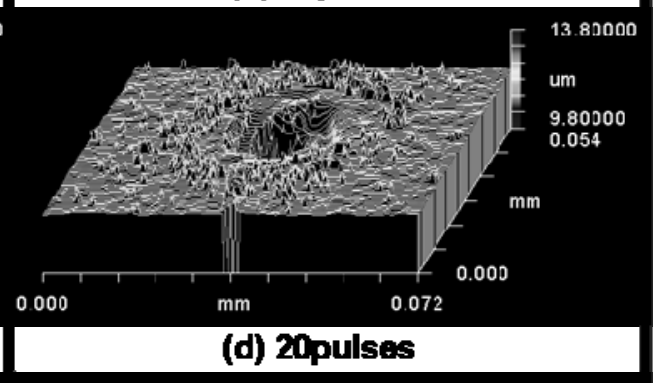

Figure 9: Changes of 3D-image at irradiated area by $266 \mathrm{~nm}$

\section{Conclusions}

Main conclusions obtained in this study are as follows.

(1) The removal depths of AlN and SiC under vacuum condition are larger compared to that under atmospheric condition regardless of laser wavelength.

(2) The severe oxidation around drilled hole was observed in the case of fourth harmonic compared to second harmonic.

(3) The surface integrity under vacuum condition was superior to that under atmospheric pressure condition, since the generation of plasma under vacuum condition was weak.

(4) In the initial stage of AlN with the fundamental wavelength, the irradiated point expanded by the absorption of laser beam with increasing the number of laser pulse, and the processing was carried out by crumbling the grain due to the thermal expansion. On the other hand, the higher harmonics could make a hole at the irradiated point by the photo-chemical reaction without thermal expansion from the initial stage.

\section{Acknowledgments}

The authors would like to thank Ibiden Co., Ltd. for supplying expensive AlN and SiC plates. The authors are also grateful to Japan Laser Corporation for their helpful assistance in experimental setup.

\section{References}

[1] N. Miyajima, 1994, New Ceramics, Chijin-shokan, pp.231 (in Japanes).

[2] O. Imanaka, 1987, Machining Handbook of Ceramics, Kensetusangyo-chousakai, pp.75 (in Japanes).

[3] S. Nakai, 1991, Laser Engineering, Ohm-sha, pp.91 (in Japanes).

[4] Mills DL, 1991, Nonlinear Optics New York springer. 